\title{
Les Etats-Unis et l'avenir du multilatéralisme
}

Guillaume Devin

\section{(2) OpenEdition}

Journals

Édition électronique

URL : http://journals.openedition.org/conflits/969

DOI : $10.4000 /$ conflits. 969

ISSN : $1777-5345$

Éditeur :

CCLS - Centre d'études sur les conflits lilberté et sécurité, L'Harmattan

Édition imprimée

Date de publication : 1 septembre 2003

Pagination : 157-174

ISBN : 2-7475-5377-9

ISSN : 1157-996X

\section{Référence électronique}

Guillaume Devin, « Les Etats-Unis et l'avenir du multilatéralisme », Cultures \& Conflits [En ligne], 51 automne 2003, mis en ligne le 02 février 2004, consulté le 30 mars 2021. URL : http://

journals.openedition.org/conflits/969; DOI : https://doi.org/10.4000/conflits.969

Ce document a été généré automatiquement le 30 mars 2021

Creative Commons License 


\title{
Les Etats-Unis et l'avenir du multilatéralisme
}

\author{
Guillaume Devin
}

La guerre américaine en Irak et la gestion de la victoire militaire ont confirmé de manière éclatante le tour unilatéral pris par la politique de l'administration du président G. W. Bush. Cette orientation a des accents radicaux, mais s'inscrit dans une longue relation d'ambivalence des Etats-Unis avec le multilatéralisme. Deux livres importants en proposent une démonstration argumentée ${ }^{1}$. La parution d'une étude française sur le débat intellectuel dans la formulation de la politique étrangère américaine complète et actualise très utilement ces analyses ${ }^{2}$. L'ensemble de ces travaux conduit à penser que la fin de la Guerre froide a accru les dilemmes de la puissance américaine et que l'engagement international des Etats-Unis, plus sélectif ou plus ouvert, déterminera largement les formes de la coopération internationale dans les années à venir.

L'existence du multilatéralisme n'est pas nouvelle : la pratique consistant à négocier à plus de trois pour définir des règles communes est connue depuis longtemps. On la retrouve dès la période classique lors de la négociation des grands traités internationaux qui vont fonder les ordres européens successifs, de la paix de Westphalie (1648) au Congrès de Vienne (1814). Néanmoins, c'est avec la création de la SDN (Société des Nations), après la Première guerre mondiale, et, plus encore, avec la création de l'Organisation des Nations Unies (ONU) en 1945 que la pratique multilatérale s'institutionnalise. Le multilatéralisme n'est plus seulement une technique diplomatique, il devient un projet politique visant à favoriser la coopération en encourageant les liens d'interdépendance entre les Etats. D'un outil relativement neutre, le multilatéralisme a été investi de vertus positives et s'est constitué comme une valeur de référence pour la conduite des affaires internationales ${ }^{3}$. Dans cette transformation, les Etats-Unis ont joué un rôle essentiel. Non seulement ils ont été à l'initiative des deux organisations mondiales (SDN et ONU), mais ils ont clairement contribué et participé à de multiples organisations internationales sectorielles dans les domaines économique, commercial, social et militaire. Pourtant les Etats-Unis n'ont pas ménagé non plus leur défiance et leurs critiques à l'égard de la coopération 
multilatérale. Pour ne s'en tenir qu'aux relations avec les deux organisations mondiales, le Sénat refusera la ratification de la Charte de la SDN et les oppositions aux Nations Unies seront fréquentes et parfois virulentes.

Cette position apparemment contradictoire n'aurait pas grande importance si les EtatsUnis n'occupaient pas une place prépondérante dans les relations internationales. Bien des Etats entretiennent des relations ambivalentes avec le multilatéralisme: tantôt prêts à jouer le jeu de la coopération multilatérale, tantôt en retrait voire en rupture avec les instances multilatérales et leurs décisions. Leurs engagements ou leurs défections ne sont pas négligeables, mais ils pèsent en proportion de leur capacité à influencer le cours des événements internationaux. Il en va de même pour les EtatsUnis dont la puissance donne, par conséquent, un tour crucial à leurs engagements, leurs non-engagements ou leurs ruptures d'engagements: si les Etats-Unis peuvent être le moteur du multilatéralisme, ils peuvent en être également le frein. Sous certains aspects (l'efficacité du multilatéralisme), on rejoint là l'hypothèse de la «théorie de la stabilité hégémonique» (hegemonial stability theory) selon laquelle la réussite d'un « régime » dépendra des capacités de l'hégémon à établir des règles, à les faire accepter par les autres Etats et à les faire respecter ${ }^{4}$. En ce sens, il est vrai qu'aucune avancée multilatérale n'est assurée (quels que soient les secteurs d'activité concernés) si les Etats-Unis ne jouent pas le jeu. Néanmoins, l'avenir du multilatéralisme ne se réduit pas aux décisions des Etats-Unis; il se jouera aussi dans les évolutions du système multilatéral lui-même et, tout particulièrement, dans sa capacité ou non à se réformer.

L'objet des quelques réflexions qui suivent concerne donc autant les positionnements américains que l'avenir du multilatéralisme.

On reviendra d'abord sur quelques facteurs pouvant expliquer l'ambiguïté récurrente des positions américaines vis-à-vis du multilatéralisme (essentiellement, ici, la SDN et l'ONU). De manière plus conjoncturelle, il conviendra ensuite de mesurer le caractère plus ou moins fluctuant des soutiens exprimés en direction des institutions multilatérales. Compte tenu de crispations plus récentes et des propres faiblesses des organisations internationales, ce sont enfin les perspectives du multilatéralisme qui seront envisagées.

Les causes durables de l'ambivalence

Trois types de facteurs se trouvent mêlés dans les phases d'engagement et de retrait des Etats-Unis vis-à-vis des institutions internationales. Ils semblent fonder une ambivalence quasi structurelle des Etats-Unis à l'égard du multilatéralisme.

L'exceptionnalisme américain

$\mathrm{Si}$ toutes les nations se pensent uniques et singulières, ce sentiment est particulièrement prononcé s'agissant du peuple des Etats-Unis. Depuis leur création, les Etats-Unis se présentent, à travers leurs représentants, comme un modèle pour le monde entier. Insistant sur l'universalité de leurs "valeurs", l'excellence de leur régime politique et leur place centrale sur l'échiquier des nations, les Etats-Unis, par la voix de leurs responsables, célèbrent depuis l'origine la grandeur matérielle et morale d'une «nation indispensable» (Bill Clinton ${ }^{5}$ ). Ce caractère prétendument exceptionnel des Etats-Unis imprègne la politique étrangère américaine et lui donne un tour particulier : il s'agit, en effet, à la fois de préserver le modèle et de le donner en exemple. Cette double inclination induit nécessairement des relations complexes avec le principe d'une concertation à plusieurs. Tantôt le retrait sera requis pour protéger le modèle tantôt l'engagement sera encouragé pour l'exporter. Mais en réalité, 
l'alternative n'est pas aussi nette. En effet, si le multilatéralisme (comme contrainte) peut entraîner des conduites de défection parce qu'il risque d'être jugé néfaste à l'intégrité du «modèle», le multilatéralisme (comme ressource) ne s'impose pas nécessairement pour promouvoir le modèle : c'est le propre de l'exceptionnalisme de fonder des discours et des pratiques messianiques qui se suffisent à eux-mêmes et qui peuvent justifier une conduite unilatérale au motif que ce qui est bon pour les EtatsUnis le serait également pour les autres pays.

On retiendra donc qu'au cours d'une histoire nationale pensée en termes d'excellence, l'engagement multilatéral n'est pas perçu comme un recours obligé : il doit servir et reste, par conséquent, assez volatile.

\section{La séparation des pouvoirs}

La définition des pouvoirs du président en matière de politique étrangère est au cœur de débats politiques et constitutionnels innombrables. Si à l'instar des autres démocraties occidentales, l'Exécutif jouit d'une prééminence dans les processus décisionnels ${ }^{6}$, le président américain est probablement plus contraint qu'ailleurs. Au titre de la Constitution, il doit ainsi composer avec les pouvoirs diplomatiques du Sénat qui sont venus, à plusieurs reprises, contrarier ses initiatives et ses engagements multilatéraux (non-ratification du Traité de Versailles en 1919, du Traité d'arms control SALT 2 en 1979 ou, plus récemment, du Protocole de Kyoto et du Traité sur l'interdiction des essais nucléaires [СТВT]). Mais, plus généralement, le président doit compter avec un Congrès attentif à ses prérogatives et à la défense de la «souveraineté nationale ». Même si à l'heure de la mondialisation, la souveraineté n'a plus qu'un sens relatif, les délégations de pouvoir à des instances multilatérales demeurent souvent perçues comme une mise en péril de la Constitution et comme une atteinte au peuple des Etats-Unis comme source ultime de la légitimité ${ }^{7}$. Parallèlement, le poids du fédéralisme et des intérêts particuliers de la vie politique américaine ne peuvent que gêner les engagements globaux et inciter les responsables (présidents et/ou élus nationaux) à la plus grande prudence vis-à-vis des organisations internationales. Les arrangements institutionnels spécifiques des Etats-Unis ne sont donc pas des plus favorables à l'engagement multilatéral. Ils ne s'y opposent pas mais le rendent toujours fragile.

Les dilemmes de la puissance

Quelle que soit la qualification que l'on retienne, les Etats-Unis se présentent comme un acteur majeur des relations internationales depuis le XIXème siècle: "grande puissance » jusqu'en 1945, l'un des deux «Super Grands » de l'ordre bipolaire jusqu'en 1989 et « puissance hégémonique » depuis. Les capacités exceptionnelles du pays dans quelques secteurs clés (économie, défense, finance, recherche) ${ }^{8}$ lui permettent de définir un bon nombre de règles du jeu international ou, à tout le moins, de ne pas se les faire imposer. Cette situation tend à faire du multilatéralisme un choix optionnel. Si les bénéfices attendus de l'engagement multilatéral ne sont pas négligeables (partage des risques, stabilité des règles, prévisibilité des conduites, légitimité), ils sont toujours mis en balance avec ses contraintes (limitations de souveraineté, atteintes à certains intérêts sectoriels, lenteur des décisions). Tous les Etats se livrent à un calcul de cette sorte, mais les Etats-Unis, plus que d'autres, ont les moyens d'explorer des solutions alternatives, bilatérales et unilatérales. C'est le privilège de la puissance d'avoir une palette d'options plus étendue. Mais c'est aussi une occasion de dilemmes lorsqu'il s'agit de savoir comment utiliser au mieux cette puissance. 
L'ambivalence des Etats-Unis à l'égard du multilatéralisme a donc des racines multiples. Il est illusoire de vouloir les isoler les unes des autres. Au mieux, on peut retenir des explications plutôt plus significatives que d'autres en fonction des cas d'espèce. Ainsi, la création et l'enthousiasme initial pour les Nations Unies ont-ils largement reposé sur l'idée que l'exceptionnalisme des Etats-Unis était appelé à exercer une influence majeure sur la définition du nouvel ordre international. Toutefois, sans le ralliement des parlementaires et les capacités concrètes mises à la disposition des Nations Unies, les ambitions de l'administration Roosevelt eurent été rapidement déçues. De même, pour prendre un exemple plus récent, si les manœuvres politiciennes entre le Congrès et la présidence sont partiellement responsables de la non-ratification du $\mathrm{CTBT}^{9}$, celle-ci peut également relever de la protection des intérêts américains compte tenu du rôle exceptionnel joué par les Etats-Unis sur la scène internationale. Les exemples pourraient être multipliés. Seul, le croisement de plusieurs facteurs explicatifs peut définir le cadre complexe dans lequel perdure et s'exprime l'ambivalence américaine à l'égard du multilatéralisme.

Les soutiens fragiles de l'engagement

Les attitudes de l'opinion publique et des élus américains sur le multilatéralisme sont relativement discordantes. Appréciées sur le moyen terme (depuis la création des Nations Unies), elles confortent l'idée que les engagements multilatéraux des EtatsUnis sont fragiles et réversibles.

Les faiblesses d'une opinion favorable

Mesurée par les sondages, l'opinion publique des Etats-Unis se présente comme globalement très favorable à la participation de leur pays aux organisations internationales ${ }^{10}$. A peu de choses près, toutes les études convergent en ce sens depuis les années 1940 . On note des périodes pendant lesquelles les opinions sont plus volatiles (les années 1970 et 1980), mais, dans l'ensemble, elles soutiennent clairement l'action des Nations Unies et la participation américaine. A la veille de l'élection du président G. W. Bush, seule une minorité soutenait le retrait des Etats-Unis des Nations Unies (15\% en 2000) tandis qu'une majorité des $2 / 3$ partageait le point de vue selon lequel les EtatsUnis devaient chercher à régler les problèmes internationaux en coopération avec les autres pays ${ }^{11}$. Cette opinion, d'autant plus positive que les enquêtés sont diplômés et dotés de revenus élevés, s'étend à l'Organisation mondiale du commerce (OMC) ou la Cour pénale internationale (CPI) et, plus généralement, à toutes les institutions multilatérales. S'agissant de l'action militaire, la préférence va également aux dispositifs multilatéraux et, parmi eux, aux Nations Unies avant l'OTAN.

En résumé, contrairement à ce que l'on pourrait croire, l'image offerte par les sondages est très nettement favorable au multilatéralisme. Il ne semble pas que les événements du 11 septembre 2001 aient modifié cette situation et tout au long des débats précédant l'intervention américaine en Irak, l'opinion publique, courtisée et fluctuante, a majoritairement penché pour une action militaire autorisée par les Nations Unies. Néanmoins, il est possible que la réussite de la campagne militaire balaie les regrets et conforte les vents de la critique. C'est en effet le paradoxe d'une opinion globalement favorable d'être également, et de manière persistante, fort sceptique sur les performances des organisations internationales. Même si le paiement des cotisations dues par les Etats-Unis aux Nations Unies est majoritairement souhaité, l'opinion, bien relayée par les élus, est toujours sensible à l'idée que les organisations internationales se prêtent aux travers de bureaucraties inefficaces. Les responsables politiques savent 
bien exploiter l'argument pour justifier, à l'occasion, leurs retraits et leurs préférences unilatérales. Les organisations internationales servent ainsi régulièrement de bouc émissaire et ceci d'autant plus facilement que le public connaît mal leur fonctionnement, leurs compétences et leurs réalisations. L'ignorance des Nations Unies par la majorité du public américain -qui ne sait pas où se situe le siège des Nations Unies ou qui confond l'UNESCO et l'UNICEF ${ }^{12}$ - est bien connue, de telle sorte que l'on peut se demander si le soutien au multilatéralisme mesuré par les sondages ne reste pas assez superficiel (l'observation ne vaut pas seulement pour les Etats-Unis). Quoi qu'il en soit, ce manque d'ancrage des positions exprimées par le public américain est une aubaine pour les décideurs qui peuvent interpréter assez librement les mouvements de l'opinion. Peu compétente, l'opinion publique livre donc un sentiment fragile qui n'est guère contraignant pour des élites beaucoup plus réservées sur les vertus du multilatéralisme.

"Politics matters"

Y a-t-il sur la question de la participation des Etats-Unis au multilatéralisme un clivage partisan clairement marqué ? Si, en général, les démocrates ont été plus favorables que les républicains, la réponse ne va pas sans nuances. Sans doute est-ce une administration démocrate qui a piloté les deux principales initiatives visant à la création d'une organisation mondiale et, par là même, encouragé les Etats-Unis à s'engager avec la SDN au lendemain de la Première guerre mondiale et avec les Nations Unies après la Seconde guerre mondiale. Pourtant, les négociations et les issues de ces deux initiatives ne se confondent pas. En effet, dans le cas du Pacte de la SDN, la nonratification vient autant de l'hostilité d'une majorité républicaine au Sénat que de l'intransigeance du président Wilson à rechercher le compromis. Si Henri Cabot Lodge (président républicain de la Commission des Affaires étrangères du Sénat) a mené une opposition vigoureuse à la ratification, il s'est également trouvé d'éminentes personnalités républicaines comme l'ancien président Howard Taft pour défendre le projet de la SDN. A contrario, tous les sénateurs démocrates n'ont pas suivi Wilson dans son refus absolu d'accepter les réserves proposées par les républicains ${ }^{13}$.

L'adoption de la Charte des Nations Unies a soulevé moins de problèmes. La crise de 1929 avait déjà souligné la nécessité de mieux organiser le système international. La guerre, la victoire de 1945 puis les tensions de la guerre froide allaient éroder les positions des isolationnistes et rallier la majorité des élites démocrates et républicaines à l'idée que les Etats-Unis avaient un rôle essentiel à jouer dans l'organisation du monde (et postérieurement dans la protection du «monde libre »). Avec le système onusien, les institutions de Bretton Woods ou les alliances militaires, c'est l'institutionnalisation de la coopération à plusieurs qui s'impose comme le principe actif de la diplomatie américaine : comme nous l'avons déjà indiqué, le multilatéralisme (le mot apparaît en 1948) n'est plus seulement un outil, il est un projet politique censé alimenter le cercle vertueux de la coopération et de la paix entre les nations en diffusant les valeurs des démocraties libérales.

Ce relatif consensus américain commença à être ébranlé lorsque les Occidentaux perdirent la majorité à l'Assemblée générale des Nations Unies. Durant la guerre de Corée, le blocage soviétique du Conseil de sécurité avait pu être contourné par un recours direct à l'Assemblée générale. Dès les années 1960, ce type de manœuvre n'est plus possible. A la grande satisfaction de l'URSS, les nouveaux Etats issus de la décolonisation multiplient les critiques à l'encontre de la politique internationale des 
Etats-Unis tandis que les débats sur le développement tournent régulièrement au désavantage des démocraties occidentales. En position défensive dans les grands forums internationaux, alors qu'ils en restent les principaux contributeurs, les EtatsUnis prennent conscience des désagréments d'un multilatéralisme qu'ils ne contrôlent qu'imparfaitement. L'irritation connaîtra une nette aggravation avec l'adoption par l'Assemblée générale des Nations Unies de la résolution 3379 (10 novembre 1975) assimilant le sionisme au racisme. A l'instar de l'ambassadeur des Etats-Unis aux Nations Unies, Daniel Patrick Moynihan, nombre de responsables américains ne considèrent plus seulement l'organisation mondiale comme un lieu de débats inefficace, mais également comme " un endroit dangereux " ${ }^{14}$. L'administration Reagan en tirera des conséquences partielles en décidant le retrait des Etats-Unis de l'UNESCO et de l'OIT, instances multilatérales jugées secondaires et trop critiques à l'égard de la politique américaine. L'ambivalence à l'égard du multilatéralisme est donc puissamment réactivée à partir des années 1970 ce dont témoignent les critiques récurrentes du Congrès à l'égard de la "bureaucratie » onusienne et sa mauvaise volonté évidente à s'acquitter des cotisations dues à l'organisation mondiale.

Malgré tout, la succession des présidents Reagan, Carter, G. H. Bush et Clinton maintiendra un «multilatéralisme de raison" résumé par la formule de Madeleine Albright: "Multilatéraux quand nous le pouvons, unilatéraux quand nous le devons $»^{15}$. La coopération internationale ne devait pas en souffrir sérieusement et la fin de la guerre froide ouvrait même des possibilités inédites d'actions communes en matière économique (évolution des missions du FMI et de la Banque mondiale), commerciale (création de l'OMC), et politico-militaire (déblocage du Conseil de sécurité et développement des opérations de maintien de la paix). Si les nuances entre républicains et démocrates demeurent perceptibles, tous les présidents penchent plutôt vers des positions conciliatrices. Le président G. H. Bush conservera ainsi une attitude prudente vis-à-vis du multilatéralisme en prenant soin de conduire la première guerre contre l'Irak sous les auspices des Nations Unies. Quant au président Clinton, il estimera que la compétitivité de l'économie et la défense des intérêts américains sont mieux servies par une participation active dans les institutions multilatérales que par une attitude de retrait.

Néanmoins, l'impression d'ensemble reste marquée par une succession de positions ad hoc. De fait, la disparition de l'URSS posait inévitablement la question du «nouvel ordre international » à construire et du rôle qu'entendait y jouer l'hégémon américain : que faire de la puissance américaine ? Comment définir les intérêts nationaux ? C'est ici que Pierre Hassner et Justin Vaïsse nous montrent de manière convaincante que le débat intellectuel et les réalignements partisans qui l'accompagnent ont été déterminants sur le cours actuel de la politique internationale des Etats-Unis. Dopés par la « victoire » de la démocratie sur le communisme et libérés de la discipline imposée par le climat de confrontation Est-Ouest, nombre de républicains et certains «transfuges » démocrates ${ }^{16}$ ont adopté des positions internationales beaucoup plus radicales et se sont démarqués à la fois des conservateurs isolationnistes (P. J. Buchanan), des réalistes classiques favorables au statu quo (H. Kissinger) et des démocrates multilatéralistes (dont l'administration Clinton était assez représentative). En ce sens, le groupe qui soutient l'élection de G. W. Bush incarne un courant assez inédit qui mêle défense de l'hégémonie américaine et unilatéralisme; désignées comme "néo-conservateurs", "réaganiens " ou "wilsoniens de droite », ces personnalités (éditorialistes comme R. Kagan ou W. Kristol ou responsables politiques comme D. Cheney, D. Rumsfeld ou P. 
Wolfowitz) sont des internationalistes, propagandistes actifs des valeurs américaines dans le monde, des " néo-impérialistes » ou des « impérialistes de la démocratie " pour lesquels le multilatéralisme est un détour largement inutile.

P. Hassner et J. Vaïsse font remonter ce courant aux années 1960 et $1970^{17}$, mais c'est avec les flottements intellectuels qui suivent la Guerre froide qu'il s'impose. A un certain pragmatisme qui domine encore les politiques des présidents $\mathrm{G}$. H. Bush et Clinton, il substitue une affirmation décomplexée de la puissance et des intérêts américains dans le monde. La victoire des républicains dans les deux chambres du Congrès en 1994 conforte ces tendances unilatéralistes et hégémonistes tandis que l'élection de G. W. Bush et les événements du 11 septembre 2001 leur donnent une occasion exceptionnelle de prospérer. La question de savoir s'il existe un moment déterminant dans ce tournant politique fait encore débat. A lire P. Hassner et J. Vaïsse, on se convainc que $\mathrm{G}$. W. Bush n'avait guère d'idée précise sur la politique étrangère au moment de son élection et que ce sont les attentats du 11 septembre qui lui ont révélé "sa mission » : la lutte contre le terrorisme ${ }^{18}$. Quoi qu'il en soit, ces événements, suivis par les victoires militaires en Afghanistan et en Irak, ont clairement profité au courant hégémoniste au détriment des réalistes classiques comme C. Powell. Si les deux lignes semblent coexister, la première a pris l'initiative. C'est là une conséquence politique importante pour une victoire électorale acquise d'extrême justesse...

L'avenir incertain du multilatéralisme

Le multilatéralisme comme vecteur privilégié de la coopération internationale est autant un choix rationnel que normatif. L'interprétation libérale qui a prévalu jusqu'à maintenant justifie la préférence accordée à ce mode d'action diplomatique en raison des avantages relatifs qu'il procurerait à tous participants et en raison de la dynamique de coopération qu'il contribuerait ainsi à alimenter. L'intérêt à coopérer multilatéralement serait récompensé par les gains de ce type de coopération. Deux idées sont donc tenues pour acquises : d'une part, le gain relatif est le choix le plus rationnel et d'autre part, la coopération de tous avec tous est la solution la plus souhaitable. Il n'est pas sûr que les Etats adhèrent de manière systématique à ces présupposés. Nombreux sont tentés par la défection au gré des opportunités lorsque les gains peuvent être plus élevés et que les risques de représailles sont limités. S'ils occupent une place importante dans la négociation (c'est-à-dire s'ils maîtrisent une part déterminante des termes de ce qui est à négocier : l'armement, le commerce, la pollution, etc.), leur défection peut être préjudiciable au fonctionnement du multilatéralisme en appauvrissant son contenu ou en réduisant sa portée. Tel est bien le problème posé par la tendance actuelle des Etats-Unis à ne plus privilégier exclusivement le cadre multilatéral. Sans qu'elle s'y réduise, la «crise du multilatéralisme» est d'abord celle provoquée par le retrait de la puissance hégémonique à l'égard d'instruments et de procédures multilatéraux (désengagement vis-à-vis de certaines conférences internationales ou des Opérations de maintien de la paix), voire son refus de s'y soumettre (non-ratification de traités) ou sa volonté de les contourner (réserves, non respect des délibérations du Conseil de sécurité ou accords bilatéraux). Cette situation a été déplorée par de nombreux analystes au nom des intérêts bien compris des Etats-Unis eux-mêmes : il serait illusoire de penser qu'il est désormais possible, même pour une grande puissance, de faire cavalier seul. Fonctionnel pour tous, le multilatéralisme serait de moins en moins un choix et de plus en plus une obligation. L'argument ne manque pas de pertinence mais il ne nous parait pas décisif. Plus exactement, il ne doit pas masquer certaines faiblesses du 
multilatéralisme qui n'est peut-être pas si «fonctionnel» que cela. A défaut d'y remédier, les positionnements critiques ne pourront que se renforcer.

\section{Les limites de l'argument fonctionnel}

Pour l'essentiel, les critiques de l'unilatéralisme de l'administration Bush (aux EtatsUnis et ailleurs) reposent sur des arguments d'efficacité et d'opportunité. Au fond, le défaut essentiel serait " $d$ 'isoler les Etats-Unis et de rendre plus difficile la poursuite de leurs intérêts $»^{19}$. On retrouve une orientation assez proche sous la plume des universitaires libéraux mais habillée de manière moins idéologique. Ici, le multilatéralisme est considéré comme inévitable : il ne se présente plus comme un choix mais comme un fait auquel les Etats ne peuvent échapper, comme une nécessité20. Comme le suggère $\mathrm{Ed}$ Luck, la question relève moins d'un jugement de valeur que d'un constat selon lequel la distance à l'égard des organisations internationales (et du multilatéralisme) est devenue " dysfonctionnelle " pour le leadership et les intérêts américains eux-mêmes ${ }^{21}$. A contrario, le multilatéralisme serait globalement fonctionnel pour le plus puissant des pays mais également pour les autres.

D'un point de vue général, il est assez difficile de trancher. L'argument fonctionnel a tendance à surestimer l'univers des contraintes internationales et par conséquent à sous-estimer celui des autonomies. S'il est vrai que l'unilatéralisme a un coût qui peut se révéler supérieur à une solution multilatérale (isolement politique, défiance des "marchés", vulnérabilité aux risques transnationaux), le multilatéralisme peut également se révéler plus lourd à supporter qu'une décision unilatérale (limitation de la souveraineté, engagement solidaire non désiré, atteinte aux intérêts catégoriels). Mais, surtout, il y a quelque chose d'un peu artificiel à confronter ces deux options comme l'on opposerait deux idéaux-types. La réalité historique est plus nuancée entre des solutions qui ne sont jamais entièrement unilatérales ou multilatérales (il existe bien des formes de multilatéralisme ${ }^{22}$ ) et qui peuvent également se combiner, pour compliquer encore un peu les choses, avec des engagements bilatéraux ou plurilatéraux. La réalité diplomatique offre du jeu et «le multilatéralisme » constitue une option, importante, certes, mais parmi d'autres.

Par ailleurs, il est indispensable d'appréhender le multilatéralisme en fonction des domaines qu'il recouvre : commerce, désarmement, environnement, droits de l'homme, etc. Certaines dimensions peuvent être plus contraignantes que d'autres : difficile de passer en force sur le terrain économique et commercial où les intérêts sont plus mêlés qu'ailleurs; mais l'échiquier militaire peut redonner une forte autonomie aux plus puissants tandis que l'échiquier des questions transnationales (migrations, environnement, terrorisme, santé, etc.) se prêtent à tous les calculs ${ }^{23}$. La situation d'ensemble est donc bien plus fluide qu'une lecture étroitement fonctionnelle pourrait le laisser croire.

Le multilatéralisme est une ressource inégale : selon les Etats et les échiquiers, il pourra être plus ou moins profitable. Avec toutes les limites d'une appréciation trop générale, on peut estimer que les Etats qui ont le plus à y gagner sont ceux qui sont assez puissants pour participer à la décision et trop faibles pour décider seuls. La coopération multilatérale sera ici, fréquemment, une condition de l'action internationale; réciproquement, la politique internationale de ces puissances dites «moyennes » sera spontanément plus favorable à la défense et à l'extension du multilatéralisme. En revanche, parce qu'ils disposent de plus grandes capacités, les Etats-Unis peuvent se permettre d'être plus sélectifs, de conserver leur leadership dans certaines instances 
multilatérales (institutions de Bretton Woods, OMC) et de pratiquer un "multilatéralisme à la carte » (Richard Haass) quand les Européens sont souvent contraints de jouer « la carte du multilatéralisme » pour se faire entendre.

Nous retrouvons là, dans la question de la puissance, un des fondements de l'ambivalence américaine vis-à-vis du multilatéralisme. Peut-être même cette ambivalence est-elle accentuée lorsque que la puissance (plus exactement, son image) est perçue comme vulnérable et menacée : méfiance et soupçons éloignent autant de la coopération multilatérale que la confiance y incite.

Quoi qu'il en soit, par leur défection, les Etats-Unis peuvent causer de sérieuses nuisances au système multilatéral. Ils peuvent en désorganiser certaines instances à la fois en réduisant les budgets et l'effectivité des décisions. Peuvent-ils pour autant détruire le multilatéralisme? Nous ne le pensons pas.

Critiques et réformes

Si l'engagement multilatéral n'est pas une nécessité, l'orientation multilatéraliste de la coopération internationale est une probabilité. La croissance du nombre des organisations internationales et le développement de leurs compétences sont désormais un fait acquis. Des avancées et des reculs sont toujours possibles, mais il paraît difficile de revenir sur ce phénomène massif et de faire comme s'il ne produisait aucun effet. La prolifération multilatérale encouragée par nombre d'Etats et confortée par les mobilisations de plus en plus fréquentes des "sociétés civiles » ont dessiné le paysage de notre modernité. Les Etats-Unis ou, plus exactement, une administration américaine à un moment donné, peuvent freiner le mouvement mais non le stopper. Dans un monde de 192 Etats, d'acteurs non gouvernementaux multiples et de réseaux transnationaux divers, des structures d'échange et de coopération sont inévitables. Les motifs de confrontation et de conflit le sont tout autant. L'ensemble pousse à des formes de concertation pour résoudre les problèmes d'intérêt commun: la "gouvernance mondiale" évoque ces différents modes de pilotage de l'action collective au plan international. Le flou de la notion a fait sa fortune ${ }^{24}$.

$C^{\prime}$ est donc moins sur le principe de la concertation internationale qu'il faut s'interroger que sur ses formes. Mais les deux questions sont indissociables. Le renforcement institutionnel est patent depuis le XIXème siècle mais il a probablement atteint certaines limites en termes d'efficacité et de légitimité. La dispersion des institutions, la contestation de leur représentativité et l'imprécision de leurs missions ont amorcé une « crise du multilatéralisme » qui ne se réduit pas aux défections américaines.

Pour retrouver ses fonctions ordonnatrices et régulatrices, le multilatéralisme doit se réformer. C'est aussi un moyen de remobiliser ses soutiens, d'y associer les mouvements de «l'opinion mondiale » et de neutraliser, autant que faire se peut, les tentations unilatérales.

On le voit, à ce stade, il n'est pas possible de disserter sur l'avenir du multilatéralisme sans adopter un biais normatif. Sans volonté de réformes, le multilatéralisme s'abîmera dans la balkanisation et la confusion. Le risque de l'éparpillement et de l'inefficacité n'est pas à exclure et il ne viendra pas tant de la puissance hégémonique que d'un système qui n'aura pas réussi à se réformer. Les grandes institutions multilatérales continueront leur(s) route(s) mais dans des logiques bureaucratiques qui favoriseront les conduites de retrait et de défection. Un nouveau souffle est nécessaire et, paradoxalement, les critiques américaines à l'égard du multilatéralisme (coûts, lenteur, dysfonctionnements, etc.) en fournissent peut-être l'occasion. 
Des propositions de réformes ont déjà été avancées ${ }^{25}$ et l'on voit assez bien les grandes orientations qui pourraient être privilégiées. (1) La représentativité politique des instances décisionnelles

Renforcer l'autorité de la gouvernance mondiale, dans le respect du pluralisme, c'est la fonder sur une représentativité élargie. Ouvrir le G7-G8, réformer le Conseil de sécurité des Nations Unies ou inventer une autre formation, plusieurs solutions sont envisageables, mais aucune ne sera satisfaisante si elle manque d'associer aux pays les plus développés, une représentation équilibrée de tous les continents à travers les puissances émergentes et les pays les plus pauvres selon des formules à négocier (membres permanents et provisoires, sélection en fonction d'indices pondérés: PNB, population, contribution).

(2) La légitimité sociale des institutions

La légitimité conforte l'autorité. En lui donnant le caractère de ce qui est juste à un moment donné, elle facilite l'adhésion et renforce la force obligatoire des règles. $\mathrm{A}$ l'aune de la référence démocratique et libérale, la représentativité politique des institutions de la gouvernance peut y contribuer mais ne suffit pas. La légitimité par les procédures (input legitimacy) et la légitimité par les résultats (output legitimacy) sont des coordonnées plus complètes de la légitimité à l'âge démocratique. La première requiert une information disponible et une transparence des modes de fonctionnement. Le droit à la critique doit pouvoir s'exercer sans difficulté et les consultations aux différents niveaux de la gouvernance (local, national, régional et mondial) doivent être étendues. Là encore, des formules sont à inventer, parfois à améliorer, notamment pour associer les acteurs non gouvernementaux (audition, expertise, forum, amicus curiae, etc.). La légitimité par les résultats suppose, pour sa part, la recherche de solutions efficaces aux problèmes identifiés : des réponses effectives aux objectifs qui sont fixés. L'instauration de mécanismes de règlement des différends, de procédures de suivi et d'évaluation, devrait être généralisée en combinant une forte implication des gouvernements dans la définition des orientations et un développement soutenu des autorités de régulation multilatérales (ARM) à la fois pour responsabiliser les premiers et pour perfectionner les secondes ${ }^{26}$.

(3) L'articulation fonctionnelle des compétences

Il faut réviser la carte du multilatéralisme. Non seulement, certaines institutions sont manquantes (sur l'environnement), mais la plupart demandent une meilleure coordination. Si l'efficacité commande de centrer les tâches sur des missions clairement délimitées, elle exige aussi une complémentarité bien ordonnée. Sous cet aspect technique perce la question politiquement délicate de la hiérarchie des préférences collectives et de la compatibilité des normes (économiques, commerciales, environnementales, sociales). A défaut de pouvoir hiérarchiser les organisations sectorielles et faute d'autorité mondiale suffisamment contraignante, il faut tendre vers la «mutualisation » des contentieux et des jurisprudences en établissant des liens procéduraux ou des passerelles plus systématiques (procédures de consultation, question préjudicielle, «avis déterminant $\aleph^{27}$ ). Le détour par le niveau régional qui procèderait déjà à une agrégation des choix pourrait faciliter les ajustements au plan mondial.

Le débat sur une nouvelle architecture institutionnelle au plan mondial est engagé. Entre les engagements sélectifs et perturbateurs de la puissance américaine et les contestations radicales de la gouvernance mondiale, la voie de la réforme est la seule 
option réaliste. Le multilatéralisme n'est pas la loi d'airain de la diplomatie moderne ; c'est une méthode qui peut défaillir parce que c'est d'abord un projet qui demande une volonté politique. Ce sont les Etats qui ont le plus à y gagner qui seront ses principaux soutiens : celles des puissances qui ne sont ni trop faibles ni trop fortes pour vouloir une coopération profitable.

\section{NOTES}

1. . Edward C. Luck, Mixed Messages. American politics and International Organization, 1919-1999, Washington DC, Brookings Institution Press, 1999, 374 p. Stewart Patrick et Shepard Forman (éds.), Multilateralism and US Foreign Policy. Ambivalent Engagement, London, Lynne Rienner Publishers, 2002, 507 p.

2. . Pierre Hassner et Justin Vaïsse, Washington et le monde. Dilemmes d'une superpuissance, Paris, Autrement, 2003, 171 p.

3. . Marie-Claude Smouts, Les organisations internationales, Paris, Armand Colin, 1995, p. 30.

4. . Sur ces approches et leurs critiques, voir Gérard Kébabdjian, Les théories de l'économie politique internationale, Paris, Editions du Seuil, 1999, notamment les chapitres 6 et 7.

5. . Cité in Edward Luck, Mixed Messages, op. cit., p. 18.

6. . Guillaume Devin, Sociologie des relations internationales, Paris, La Découverte, 2002, pp. 50-55.

7. . Edward Luck, Mixed Messages, op. cit., pp. 30-31.

8. . Cette " puissance structurelle » (S. Strange), entendue ici au sens large, combine à la fois les atouts du hard power (capacité de contraindre) et ceux du soft power (capacité de convaincre) ; sur la notion de puissance structurelle, Susan Strange, « Toward a Theory of Transnational Empire » in Ernst-Otto Czempiel et James N. Rosenau (éd.), Global Changes and Theoretical Challenges, Lexington, Lexington Books, 1989, pp. 161-176 et sur la distinction entre hard power et soft power, voir Joseph S. Nye, Le leadership américain. Quand les règles du jeu changent, Nancy, Presses universitaires de Nancy, 1992.

9. . Terry L. Deibel, « The Death of a Treaty », Foreign Affairs, vol.81, n5, septembreoctobre 2002, pp. 142-161.

10. . Edward Luck, Mixed Messages, op. cit., pp.260-279 et Steven Kull, « Public Attitudes Toward Multilateralism » in Stewart Patrick and Shepard Forman (éd.), Multilateralism and US Foreign Policy, op. cit., pp. 99-120.

11. . Steven Kull, Ibid, pp.100-101.

12. . Edward Luck., Mixed Messages, op. cit., p. 264.

13. . Charles Zorgbibe, Wilson. Un croisé à la Maison-Blanche, Paris, Presses de Sciences Po, 1998, pp. 363-373.

14. . Cité in Edward Luck, Mixed Messages, op. cit., p. 114

15. . Cité in Pierre Hassner et Justin Vaïsse, Washington et le monde, op. cit., p. 75.

16. . Ibid, p. 27. 
17. Ibid, pp. 26-28.

18. . Ibid, p. 86.

19. . Patrick Allard, « Les Etats-Unis et la gouvernance mondiale », Conseil d'Analyse Economique (CAE), La gouvernance mondiale, Paris, La Documentation Française, 2002, p. 260.

20. . Shepard Forman, « Multilateralisme as a Matter of Fact : US Leadership and the Management of the International Public Sector » in Stewart Patrick and Shepard Forman (éd.), Multilateralism and US Foreign Policy, op. cit., p. 439.

21. . Edward Luck, Mixed Messages, op. cit., p. 12.

22. . Miles Kahler, « Multilateralism with Small and Large Numbers » in John Gerard Ruggie (éd.), Multilateralism Matters, New York, Columbia University Press, 1993, pp. 295-326.

23. Sur cette approche d'un échiquier international en trois dimensions, voir Joseph Nye, The Paradox of American Power - Why the World's Only Super-power Can't Go It Alone, New York, Oxford University Press, 2002 .

24. . Marie-Claude Smouts, « La coopération internationale : de la coexistence à la gouvernance mondiale » in Marie-Claude Smouts (dir.), Les nouvelles relations internationales. Pratiques et théories, Paris, Presses de Sciences-Po, pp. 135-160.

25. . Dans le cas français, voir Pierre Jacquet, Jean Pisany-Ferry et Laurence Tubiana, « Rapport de synthèse du Conseil d'Analyse Economique » (CAE), Gouvernance mondiale, Paris, La Documentation Française, 2002, pp. 9-117. Voir également les travaux de Robert $\mathrm{O}$. Keohane et Joseph S. Nye, "Governance in a globalizing world » et « The club model of multilateral cooperation and problems of democratic legitimacy » in Robert O. Keohane, Power and Governance in a Partially Globalized World, Londres, New York, Routledge, 2002, pp. 193-218 et pp. 219-244.

26. . Sur les ARM, voir le « Commentaire » d'Elie Cohen au rapport de synthèse du CAE, Gouvernance mondiale, op. cit., pp. 127-143.

27. . Sur ces solutions procédurales, voir Marie-Anne Frison-Roche, « Le droit, source et forme de régulation mondiale », ibid., pp. 313-330 et Bernard Grelon, « Regard juridique sur la hiérarchie des normes ", ibid., pp. 331-348.

\section{INDEX}

Thèmes : Nations Unies (ONU)

Mots-clés : diplomatie, droit international, normes internationales, organisations internationales, politique étrangère, Relations Internationales

Index géographique : Etats-Unis 